\title{
Neoplasia oncocítica adrenocortical de potencial maligno incierto: reporte de un caso
}

\author{
Santiago Beltrán-Flores ${ }^{1}$, Alejandra Razzeto ${ }^{2}$, Yolanda Scavino-Levy ${ }^{3}$ y Carlos Santa María ${ }^{4}$
}

'Departamento de Cirugía General, Clínica Angloamericana. Lima, Perú Departamento de Medicina Interna, Clínica San Felipe. Lima, Perú ${ }^{3}$ Departamento de Patología Clínica Angloamericana. Lima, Perú

4Departamento de Urología Clínica Angloamericana. Lima

Recibido el 11 de octubre de 2108 y aceptado para publicación el 23 de enero de 2019.

Correspondencia a: Dr. Santiago Beltran-Flores beltransantiago03@gmail.com

\section{Adrenocortical oncocytic neoplasm with uncertain malignant potential:} a case report

Objective: To describe a case report of a oncocytic adrenocortical neoplasm, treated surgically in a clinic in Lima, Peru. Case report: A 26-year-old woman is admitted to the emergency due to nonspecific abdominal pain. A tumor measuring $15 \times 14 \times 12 \mathrm{~cm}$ dependent on left adrenal gland is evidenced, so surgical treatment is decided. Pathological analysis evidences oncocytic neoplasia of uncertain malignant potential. Discussion: Oncocytic adrenocortical neoplasms are rare entities, with few case reports of this disease. To classify them, the modified Weiss scale is used. We obtain an oncocytic neoplasm of uncertain malignant potential, whose treatment includes surgery for tumor resection and observation. Conclusion: Consider oncocytic neoplasms within the differential diagnosis of adrenal incidentalomas.

Key words: adrenal incidentaloma; oncocytoma; neoplasia.

\section{Resumen}

Objetivo: Describir el caso clínico de una paciente con neoplasia oncocitica adrenocortical, tratado quirúrgicamente en una clínica de Lima, Perú. Caso clínico: Paciente mujer de 26 años ingresa a emergencia por dolor abdominal inespecífico. Se evidencia tumoración de $15 \times 14 \times 12 \mathrm{~cm}$ dependiente de glándula suprarrenal izquierda por lo que se decide tratamiento quirúrgico. Al análisis patológico se evidencia neoplasia oncocítica de potencial maligno incierto. Discusión: Las neoplasias oncocíticas adrenocorticales son entidades poco frecuentes, con escasos reportes de casos de esta enfermedad. Para clasificarlas, se usa la escala de Weiss modificada. Obtenemos una neoplasia oncocítica de potencial maligno incierto, cuyo tratamiento incluye la cirugía de resección de tumor y observación. Conclusión: Considerar a las neoplasias oncocíticas dentro del diagnóstico diferencial de incidentalomas adrenales.

Palabras clave: incidentaloma adrenal; oncocitoma; neoplasia.

\section{Introducción}

Los tumores de la corteza suprarrenal son entidades cuya incidencia han ido en aumento, probablemente, debido a un mayor uso de estudio por imágenes y al mejoramiento técnico de éstas ${ }^{1}$. Dentro de las neoplasias de la corteza suprarrenal encontramos a los oncocitomas, estos tumores se originan principalmente en riñón, tiroides, paratiroides y timo ${ }^{2}$.

A pesar que las neoplasias de esta estirpe celular están bien identificadas en dichos órganos, solo existen escasos reportes de tumores oncocíticos provenientes de la corteza de la glándula suprarrenal, habiendo revisiones sistémicas que llegan hasta máximo 150 pacientes. Estas patologías ge- neralmente son de carácter benigno y el tratamiento quirúrgico usualmente es curativo ${ }^{3}$.

Presentamos un caso de esta rara entidad que se presentó en el servicio de cirugía de nuestro centro.

\section{Caso clínico}

Paciente mujer de 26 años ingresa al servicio de emergencia por dolor abdominal difuso posterior a ingesta copiosa de alimentos. No presenta antecedentes médicos de importancia. El examen físico revela dolor ligero a nivel de hipocondrio derecho, no se palpan masas ni resistencia muscular. Se realizan estudios ecográficos para descartar enfermedad 
biliar aguda. La ecografía abdominal no evidencia patología biliar, sin embargo, se evidencia una gran tumoración a nivel retroperitoneal por lo que sugiere ampliar estudios.

Se decide internación, solicitándose tomografía abdominal que evidencia una masa retroperitoneal izquierda de $14 \mathrm{~cm}$ probablemente dependiente de corteza suprarrenal (Figura 1). En los exámenes hormonales, se encuentran ACTH plasmática, electrolitos y metanefrinas en orina en valores normales, por lo que se descarta una masa funcional. Se decide

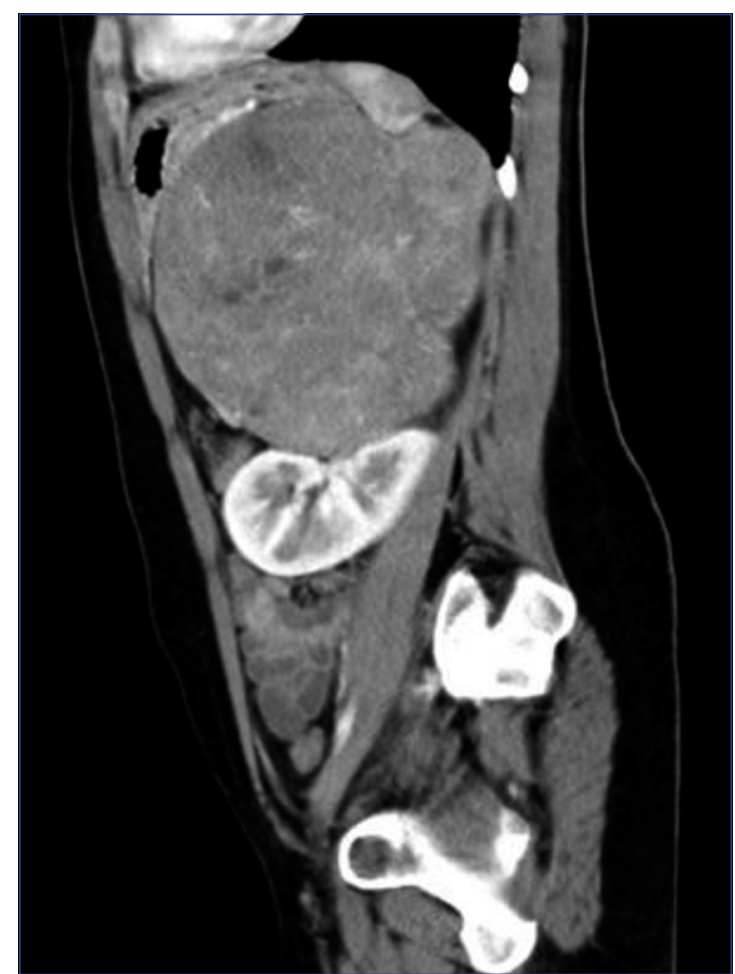

Figura 1. Masa retroperitoneal que reemplaza glándula suprarrenal.

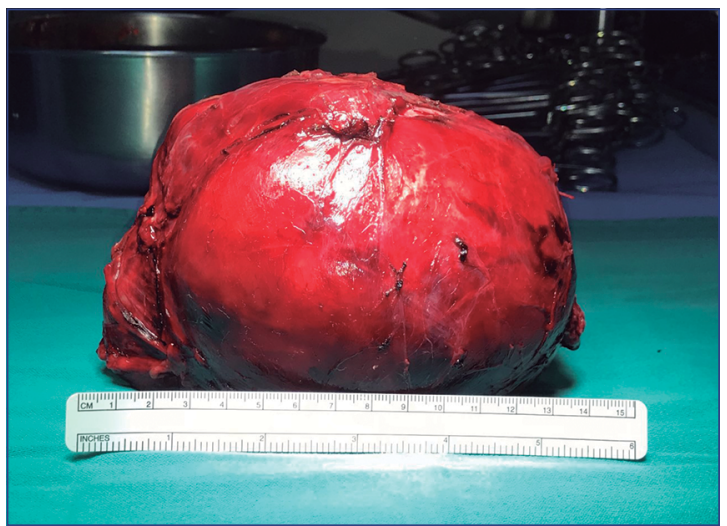

Figura 2. Tumoración adrenal de $15 \times 14 \times 12 \mathrm{~cm}$. realizar una adrenalectomía izquierda con extirpación tumoral como tratamiento y estudio.

En sala de operaciones se evidencia una tumoración de $15 \times 14$ x $12 \mathrm{~cm}$ aparentemente proveniente de la glándula suprarrenal izquierda, encapsulada que comprime bazo, pero no lo invade, no se evidencian metástasis (Figura 2). Al análisis microscópico, se evidencian células eosinofílicas granulares informadas como oncocíticas (Figuras 3 y 4). Al

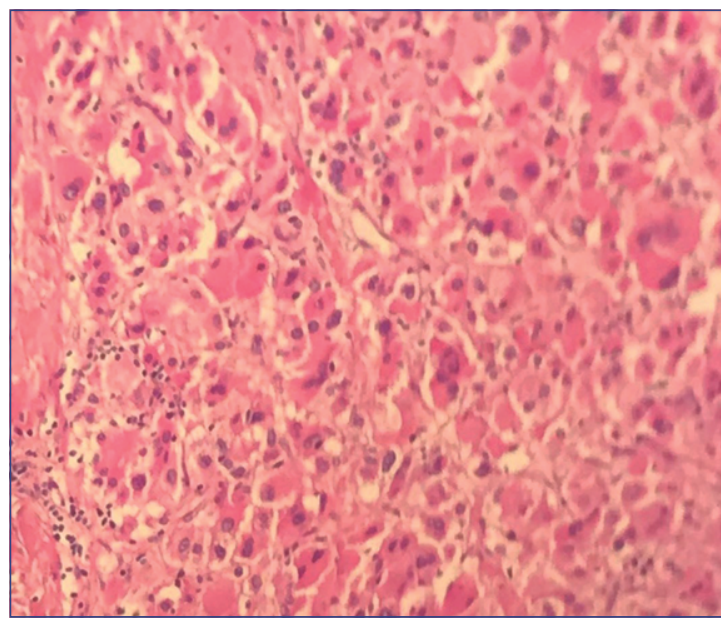

Figura 3. Zona de atipia celular

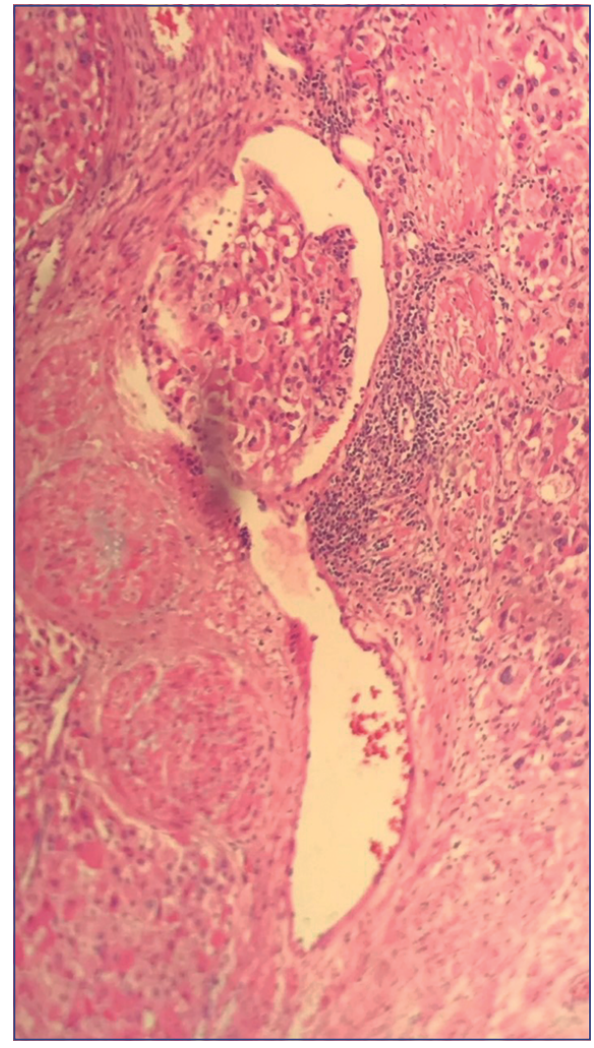

Figura 4. Sospecha de invasión vascular. 
ampliar el estudio se encuentran los siguientes datos: actividad mitótica $<1 / 50 \mathrm{HPF}$, presencia de necrosis e infiltración capsular parcial sin sobrepasarla. La inmunohistoquímica revela Ki-67 1-2\%, Melan A positivo y cromogranina negativo, el examen con reticulina no evidencia disrupción de la trama.

Se realiza el diagnóstico de neoplasia adrenal oncocítica de potencial maligno incierto, la paciente evoluciona favorablemente en el post operatorio y es dada de alta para realizar seguimiento por consultorio externo.

\section{Discusión}

El caso se presenta como una masa adrenal incidental, es decir, un incidentaloma adrenal. La prevalencia de los incidentalomas adrenales ha ido en aumento con el aumento del uso de exámenes por imágenes ${ }^{1}$. La investigación de estas entidades debe incluir pruebas de laboratorio para evaluar funcionalidad y un correcto estudio imagenológico ${ }^{4,5}$. Pese a que las características tomográficas de los tumores retroperitoneales pueden predecir su potencial maligno, en caso de las neoplasias oncocíticas no se ha demostrado dicha relación, por lo que el mejor predictor es el análisis patológico ${ }^{6}$.

El tratamiento indicado en casos de masas suprarrenales mayores a $4 \mathrm{~cm}$ incluye la adrenalectomía total $^{4}$. En el caso presentado se obtiene una masa de $14 \mathrm{~cm}$ que no invade órganos adyacentes por lo que se realiza adrenalectomía total. El análisis patológico evidencia una neoplasia oncocítica dependiente de glándula suprarrenal.

Dentro de los casos reportados en la literatura, se tiene que la mayoría de estos se presentan como masas asintomáticas, excepto por limitadas ocasiones en las que se presentan como causa de dolor abdominal ${ }^{7}$. Con respecto a edad y sexo, no se ha observado una distribución homogénea en edad, con mayor frecuencia en mujeres y generalmente posicionado en zona izquierda ${ }^{8}$.

Tradicionalmente, se ha usado la escala de Weiss para valorar el potencial maligno de las neoplasias cortico adrenales ${ }^{9}$, sin embargo, el comportamiento de las neoplasias oncocíticas no sigue el patrón obtenido por este score, es por eso que Bisceglia y cols realizaron una modificación a dicha escala para neoplasias oncocíticas cortico adrenales ${ }^{10}$. Según esta escala, tenemos que nuestro caso se encasilla en un potencial maligno incierto.

Además del uso de la escala modificada de Weiss, se han usado otros parámetros para predecir el comportamiento maligno de estas neoplasias. Estos incluyen el uso de Ki-67 y el uso del algoritmo de reticulina ${ }^{11,12}$, que en nuestro caso se encuentra dentro de parámetros normales. El tratamiento sugerido en estos casos es la adrenalectomía total preferentemente por laparotomía, aunque, si es técnicamente posible, se puede usar la vía laparoscópica ${ }^{8}$. En los casos reportados, las neoplasias oncocíticas adrenales de potencial maligno incierto presentan tasas de recurrencias escasas ${ }^{13}$, sugiriéndose evaluación periódica con tomografías abdominales y pruebas de funcionalidad ${ }^{8}$.

\section{Conclusión}

Considerar las neoplasias oncocíticas corticoadrenales dentro del diagnóstico diferencial de incidentalomas de glándula suprarrenal. Se recomienda realizar un estudio profundo tanto patológico como inmunohistoquímico para poder realizar una clasificación correcta. De acuerdo a la literatura observada, en caso de neoplasia de potencial maligno incierto se recomienda tratamiento quirúrgico y observación mediante técnicas de imagen y exámenes hormonales.

\section{Responsabilidades éticas}

Protección de personas y animales. Los autores declaran que para esta investigación no se han realizado experimentos en seres humanos ni en animales.

Confidencialidad de los datos. Los autores declaran que han seguido los protocolos de su centro de trabajo sobre la publicación de datos de pacientes.

Derecho a la privacidad y consentimiento informado. Los autores declaran que en este artículo no aparecen datos de pacientes.

Conflictos de interés: No hay. 


\section{Bibliografía}

1. Bovio S, Caraldi A, Reimondo G, Sperone P, Novello S, Berruti A, et al. Prevalence of adrenal incidentaloma in a contemporary computerized tomography series. J Endocrinol Invest. 2006;29:298302.

2. Lyu L, Wang Q, Song S, Li L, Zhou H, Li M, et al. Oncocytic tumors are marked by enhanced mitochondrial content and mtDNA mutations of complex I in Chinese patients. Mitochondrion 2018;pii:S1567-7249(17)30335-5.

3. Harbin AC, Chen A, Bhattacharyya S, Khurana JS, Kaplan JR, Eun DD. Oncocytic Adrenocortical Neoplasm Diagnosed after Robot-Assisted Adrenalectomy. Case Rep Urol. 2015;2015:515071.

4. Fassnacht M. Management of adrenal incidentalomas: European Society of Endocrinology Clinical Practice Guideline in collaboration with the European
Network for the Study of Adrenal Tumors Eur J Endocrinol. 2016;175:G1-G34.

5. Zeiger M. American Association of Clinical Endocrinologists and American Association of Endocrine Surgeons Medical Guidelines for the Management of Adrenal Incidentalomas. Endocrine Practice 2009;15:1-20.

6. Tirkes T, Gokaslan T, McCrea J, Sandrasegaran K, Hollar M, Akisik F, et al. Oncocytic Neoplasms of the Adrenal Gland. American Journal of Roentgenology 2011;196:592-6.

7. Hoang MP, Ayala AG, Albores-Saavedra J. Oncocytic adrenocortical carcinoma: a morphologic, immunohistochemical and ultrastructural study of four cases. Mod Pathol. 2002;15:973-8

8. Mearini L, Del Sordo R, Costantini E, Nunzi E, Porena M. Adrenal oncocytic neoplasm: a systematic review. Urol Int. 2013;91:125-33

9. de Krijger RR, Papathomas TG. Adrenocortical neoplasia: evolving concepts in tumorigenesis with an emphasis on adrenal cortical carcinoma variants. Virchows Arch. 2012;460:9-18.

10. Bisceglia M, Ludovico O, Di Marria A, Ben-Dor D, Sandbank J, Pasquinelli G, et al. Adrenocortical oncocytic tumors: report of 10 cases and review of the literature. Int J Surg Pathol. 2004;12:23143.

11. Libé R. Adrenocortical carcinoma (ACC): diagnosis, prognosis, and treatment. Frontiers in Cell and Developmental Biology 2015;3:45.

12. Duregon E, Fassina A, Volante M, Nesi G, Santi R, Gatti G, et al. The reticulin algorithm for adrenocortical tumor diagnosis: a multicentric validation study on 245 unpublished cases. Am J Surg Pathol. 2013;37:1433-40.

13. Lee CK, Choi KH, Cha YJ, Bum JH, Lee HH, Kim MS, et al. Large Oncocytic Adrenocortical Tumor with Uncertain Malignant Potential. Korean Journal of Urology 2011;52:650-2. 